\title{
Co-existence and shell structures of several superfluids in trapped three-component Fermi mixtures
}

\author{
T. Paananen, ${ }^{1}$ P. Törmä, ${ }^{2}$ and J.-P. Martikainen ${ }^{1}$ \\ ${ }^{1}$ Department of Physical Sciences, University of Helsinki, \\ PO Box 64, 00014 University of Helsinki, Finland \\ ${ }^{2}$ Nanoscience Center, Department of Physics, PO Box 35, 40014 University of Jyväskylä, Finland
}

(Dated: September 23, 2018)

\begin{abstract}
We study the properties of a trapped interacting three component Fermi gas. We assume that one of the components can have a different mass from the other two. We calculate the different phases of the three component mixture and find a rich variety of different phases corresponding to different pairing channels, and simple ways of tuning the system from one phase to another. In particular, we predict co-existence of several different superfluids in the trap, forming a shell structure, and phase transitions from this mixture of superfluids to a single superfluid when the system parameters or temperature is varied. Such shell structures realize superfluids with a non-trivial spatial topology and leave clear observable signatures in the density profile of the gas.
\end{abstract}

The observation of fermion pairing [1, 2, 3, 4, 5] and quantized vortices [6] in ultracold Fermi gases have opened up new opportunities for exploring many-body quantum physics. For example, these systems can be used to study also strongly interacting fermionic superfluids with mismatched Fermi surfaces [7]. Experiments with such trapped polarized Fermi gases have revealed exciting and non-trivial phase separation properties [8, 9, 10]. These system have some intriguing similarities with electronic systems where external magnetic field imposes unequal mixture of electron spins as well as with quark matter systems where color superconductivity may appear [11]. In color superconductivity mismatched Fermi surfaces are due to unequal quark masses and such phenomena might be realized in the core of the compact star or in low-energy heavy-ion collisions. There is no fundamental problem in trapping and cooling also atoms with different masses and indeed both boson-boson as well as boson-fermion mixtures composed of different atoms have been already experimentally realized [12, 13] and several groups are currently working towards the creation of different fermion-fermion mixtures. The prospect of degenerate fermion mixtures with unequal masses has naturally motivated also recent theoretical contributions [14, 15, 16, 17]. In this article we present general phase diagrams for a trapped threecomponent mixture of interacting fermions and predict, for instance, co-existence of several superfluids and superfluid shell-structures.

Some studies related to three-component Fermi gases have been done [18, 19, 20], but these studies were restricted either to an unlikely symmetric setup [18], to equal masses 19], or additional simplifying assumptions were made concerning the strength of the various couplings in the system [19, 20]. Future experiments will certainly be done in an external trap and when the numbers of particles are different, the trapped physics can be quite different from the physics in a uniform system. For this reason we will not here only allow different masses and varying coupling strengths in different pairing channels, but will also solve the realized superfluid states in a trap. When interactions in all possible pairing channels are included, the number of trapped phases can become very high. For example, part of the trap might be occupied by one type of superfluid, while some other part of the trap is occupied by a different superfluid or by a normal state. Even though the number of possible configurations at first appears daunting, using the local density approximation, we find a simple picture which explains most of the trapped phases. As it turns out, one can experimentally tune the system from one phase to another, by changing either the strength of interactions, trapping frequency, atom number, or temperature.

We assume here that the third component has a different mass from the other two components. Consequently, as a by product, we also solve the trapped physics of the unequal mass two-component Fermi mixtures. Furthermore, we briefly discuss the strength of induced interaction as the mass ratio is varied and find that the role of induced interactions becomes more important as the mass difference increases.

We employ generalization of the BCS mean-field theory which is expected to be quantitatively fairly reliable in the weak coupling limit or with stronger interactions at zero temperature. At non-zero temperatures mean-field theory gives qualitatively reasonable results, but many body effects can alter the critical temperature substantially. Our Hamiltonian is given by

$$
\begin{gathered}
\hat{H}=\int d \mathbf{r} \sum_{\sigma=1,2,3}\left(-\frac{\hbar^{2} \nabla^{2}}{2 m_{\sigma}}+V_{\sigma}(\mathbf{r})-\mu_{\sigma}\right) \hat{\psi}_{\sigma}^{\dagger}(\mathbf{r}) \hat{\psi}_{\sigma}(\mathbf{r})+ \\
\Delta_{12} \hat{\psi}_{1}^{\dagger}(\mathbf{r}) \hat{\psi}_{2}^{\dagger}(\mathbf{r})+\Delta_{12}^{*} \hat{\psi}_{2}(\mathbf{r}) \hat{\psi}_{1}(\mathbf{r})+\Delta_{13} \hat{\psi}_{3}^{\dagger}(\mathbf{r}) \hat{\psi}_{1}^{\dagger}(\mathbf{r}) \\
+\Delta_{13}^{*} \hat{\psi}_{1}(\mathbf{r}) \hat{\psi}_{3}(\mathbf{r})+\Delta_{23} \hat{\psi}_{3}^{\dagger}(\mathbf{r}) \hat{\psi}_{2}^{\dagger}(\mathbf{r})+\Delta_{23}^{*} \hat{\psi}_{2}(\mathbf{r}) \hat{\psi}_{3}(\mathbf{r}) \\
-\left|\Delta_{12}\right|^{2} / g_{12}-\left|\Delta_{13}\right|^{2} / g_{13}-\left|\Delta_{23}\right|^{2} / g_{32}
\end{gathered}
$$

where $\hat{\psi}_{\sigma}^{\dagger}(\mathbf{r})$ and $\hat{\psi}_{\sigma}(\mathbf{r})$ are fermionic creation and annihilation operators of the $\sigma$-component and interaction strengths are given by $g_{\sigma \sigma^{\prime}}=2 \pi \hbar^{2} a_{\sigma \sigma^{\prime}} / \mu_{r}$, where $a_{\sigma \sigma^{\prime}}$ 
is the scattering length and $\mu_{r}=\left(1 / m_{\sigma}+1 / m_{\sigma^{\prime}}\right)^{-1}$ is the reduced mass of the scattering atoms.For concreteness, most of our calculations are applied to a situations in which two of the components are different hyperfine states of ${ }^{6} \mathrm{Li}$ atoms and one of the components consists ${ }^{40} \mathrm{~K}$ atoms, although we also draw some general conclusions with arbitrary mass ratios. This implies $m_{1}=m_{2}$. $\Delta_{\sigma \sigma^{\prime}}(\mathbf{r})=g_{\sigma \sigma^{\prime}}\left\langle\hat{\psi}_{\sigma}(\mathbf{r}) \hat{\psi}_{\sigma^{\prime}}(\mathbf{r})\right\rangle$ are the order parameters which we find by minimizing the grand canonical potential $\Omega=-k_{B} T \ln \operatorname{Tr}\left[e^{-\beta \hat{H}}\right]$. Here the $k_{B}$ is Boltzmann constant, $T$ is the temperature, and $\beta=1 / k_{B} T$. Unless indicated otherwise, we use the unit of length $1 / k_{F}$, where $k_{F}$ is the Fermi momentum of the first component ideal gas in the center of the trap. Due to the use of contact interaction some integrals in momentum space diverge and are regularized, as usual, by subtracting the divergent contribution away. In this article we will focus on the most interesting case when all coupling strengths are negative.

Here we take the trapping potentials to be harmonic $V_{\sigma}(\mathbf{r})=m_{\sigma} \omega_{\sigma}^{2} r^{2} / 2$. The potentials are identical for the same mass atoms, which is accurate for the lowest two hyperfine states of ${ }^{6} \mathrm{Li}$, for example, but the heavy atoms are assumed to experience a different trapping potential. This is in general true and having identical trapping potentials would require extra effort. In order for the details of our calculations to be valid, the heavy fermion should nevertheless experience a trap with a same aspect ratio as the light fermions. Most of our results will nevertheless remain qualitatively valid even with more complicated trapping scenarios.

In order to compute different configurations we

employ a local density approximation (LDA). In LDA we can, at each point in space, use a local chemical potential $\mu_{\sigma}(\mathbf{r})=\mu_{\sigma}-V_{\sigma}(\mathbf{r})$ and find the state which minimizes the grand potential. The chemical potentials in the center of the trap must then be determined in such a way that atom numbers have desired values. LDA could also be used in a more refined way by minimizing energy over the whole system [21] while including the possibility of more exotic superfluid phases such as the breachpairing/Sarma state.

Trapped phases. - The minimization of the grand potential reveals that in a uniform system one has four different phases, three different superfluid phases corresponding to different pairing channels with the remaining component in a normal state, and a normal Fermi gas. We find some general rules in the weak coupling regime which predict the phase which is energetically favorable at zero temperature. First, pairing will occur only for closely matched Fermi surfaces. How closely the surfaces must be matched depends on the coupling strengths and stronger coupling implies greater tolerance for a Fermi surface mismatch. Second, if several pairing channels have same coupling strengths the pairing will occur in the channel with involving the heavy fermion. This ef- fect is due to the density of states increasing with mass which translates into lower energy. However, it should be kept in mind that this density of states effect can be masked by increasing the coupling in the channel involving the light fermion.

In the trap, the situation easily becomes more rich. The variables one can easily vary independently are the particle numbers of each component $N_{\sigma}$, ratio of the trapping frequencies for ${ }^{40} \mathrm{~K}$ and ${ }^{6} \mathrm{Li}$ atoms, $T$, and using a (wide) Feshbach resonance, at least one of the coupling strengths. As we demonstrate in Fig. 1 at $T=0$, one can easily find examples of systems with all four different phases co-existing in the trap at the same time. This is made possible by unequal atom numbers and different trapping frequency of the ${ }^{40} \mathrm{~K}$-component.

The Fermi surfaces are matched when local chemical potentials satisfy $\mu_{\sigma}(\mathbf{r})=m_{\sigma} / m_{\sigma^{\prime}} \mu_{\sigma^{\prime}}(\mathbf{r})$. When the numbers of atoms in the equal mass 12-channel are different also their chemical potentials in the center of the trap are different and their Fermi surfaces are not sufficiently matched throughout the system. For a two component system this would result in a superfluid core surrounded by a normal gas $[8,9,10,22]$, but in an interacting three component system superfluidity in the two unequal mass pairing channels can appear. Since the number of atoms and trapping frequencies are different, Fermi surfaces can be matched for different pairing channels at different locations in the trapped gas. The requirement of matched Fermi surfaces implies that it is most natural to define a dimensionless effective trap frequency for the third component through $r_{\omega}=m_{\mathrm{K}} \omega_{\mathrm{K}} / m_{\mathrm{Li}} \omega_{\mathrm{Li}}$. When $r_{\omega}=1$ the Fermi surfaces which are matched at the origin remain matched throughout the trap. For concreteness we assume a harmonic trap for Lithium atoms with a geometric average of trap frequencies $\omega_{\mathrm{Li}}=670 \mathrm{~Hz}$

Since it is experimentally easier to observe integrated densities as opposed to a three-dimensional density distributions, in part (b) of Fig. 1 we show the doubly integrated density differences $\Delta n_{\sigma \sigma^{\prime}}(z)=\int d x d y n_{\sigma}(\mathbf{r})-$ $n_{\sigma^{\prime}}(\mathbf{r})$. We find that superfluid regions appear as flat areas in the integrated density-differences. If the number of atoms participating in the superfluidity in some channel is high enough, it can leave behind a clearly observable experimental signature in the density distributions, not unlike what happens in a polarized two-component systems [10, 22].

Let us now discuss the dependence of the system on experimentally easily tunable parameters. In Fig. 2 (a) we show the phase diagram as a function of the effective frequency ratio $r_{\omega}$ and position in a trap. For equal effective trapping the system is a pure 23-superfluid, due to the higher density of states for the unequal mass channel. The area occupied by the 23 -superfluid becomes smaller with increasing $r_{\omega}$ and contracts to a shell surrounded by 12 -superfluids. This is caused by the reduction in the area of sufficiently well matched Fermi surfaces with in- 
creasing $r_{\omega}$.

Fig. 2 (b) shows the phase diagram as a function of the particle number ratio $N_{3} / N_{2}$. Because we choose $r_{\omega}=1, \mu_{\sigma}(\mathbf{r})$ profiles have the same shape for all three components and one can get matched Fermi surfaces and superfluidity in the 23-channel only if the density of the third component is high enough in the center of the trap. For this reason there is no superfluid state $\Delta_{23}$ for small number of $N_{3}$ atoms. Similar conclusion applies if the number of atoms in the third component is excessively high.

In Fig. 3 (a) we show the phase diagram in the trap as a function $-k_{F} a_{12}$. We find that when one approaches the Feshbach resonance and $-k_{F} a_{12}$ becomes large there is phase transition from the superfluid state $\Delta_{23}$ to the superfluid state $\Delta_{12}$. In this case there are two competing two component systems. As the interaction in the same mass channel increases, pairing in that channel eventually becomes energetically favorable even if the average mass in that channel is lower. In Fig. 3 (b) we demonstrate the phase diagram in the trap as a function $-k_{F} a_{12}$, when the trapping potentials are different. When $k_{F} a_{12} \in[-0.35,-0.2]$ we find, an unusual configuration, a normal gas in the center of the trap and a shell of 23-superfluid state surrounding it. This can be understood by the fact that pairing with the third component modifies the density distribution of the components so much that the Fermi surfaces of the first and second components do not match in the center of the trap anymore. A BCS solution is possible, at zero temperature, only when $\left(\mu_{1}-\mu_{2}\right) / 2<\Delta_{12}$ and since the gap $\Delta_{12}$ would be small for small $\left|k_{F} a_{12}\right|$, modifications of density distributions do not have to be dramatic to make the BCS solution disappear in the center of the trap.

The mean-field critical temperature for superfluidity between atoms with unequal masses in the BCS regime can be calculated and is given by $k_{B} T_{c}=$ $\left[8 \gamma \sqrt{r_{m}} /\left(e^{2} \pi\right)\right] \epsilon_{F} \exp \left[\pi /\left(2\left|k_{F} a\right|\right)\right]$, where $r_{m}=m_{\mathrm{Li}} / m_{\mathrm{K}}$. Therefore, for the same value of the scattering length the $T_{c}$ for a system with unequal masses is changed by a factor of $\sqrt{r_{m}}$ from the equal mass case. On the other hand at $T=0$ pairing in the unequal mass channel is energetically favored due to higher density of states of heavy fermions. Therefore, there is an intermediate (2nd order) transition from the unequal mass channel to the same mass channel. This is demonstrated in Fig. 4 where we show the phase diagram as of function of $T$. One clearly sees that as the temperature rises there is first a transition between the pairing channels, which is then followed by a transition into a normal state. In a trap, different pairing channels can again coexist in some intermediate temperature window.

Many-body effects due to scattering in a medium modify interactions between fermions and can substantially lower the critical temperature. This correction due to induced interactions was calculated by Gorkov and Melik- (a)

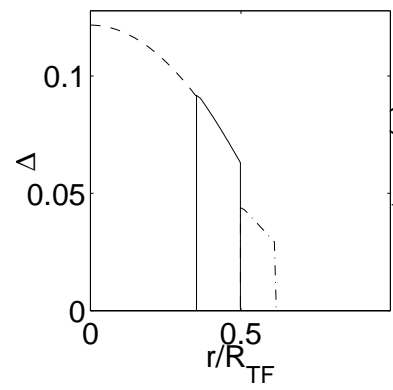

FIG. 1: (a) Gaps as a function of position $\left(R_{T F}\right.$ is the ideal gas Thomas-Fermi radius of the second component). Solid, dashed, and dashed-dotted lines describe $\Delta_{23}, \Delta_{13}$, and $\Delta_{12}$ respectively. (b) Doubly integrated density differences. Solid, dashed, and dashed-dotted lines describe $\Delta n_{23}, \Delta n_{13}$, and $\Delta n_{12}$ respectively. We used the parameters $N_{1}=6 \cdot 10^{4}, N_{2}=$ $5 \cdot 10^{4}$, and $N_{3}=2 \cdot 10^{4}, k_{F} a_{12}=-1.04,\left(1+1 / r_{m}\right) k_{F} a_{13}=$ -1.03, $\left(1+1 / r_{m}\right) k_{F} a_{23}=-1.15$, where $r_{m}=m_{L i} / m_{K}$ and $\omega_{3} / \omega_{1}=0.4$.

(a)
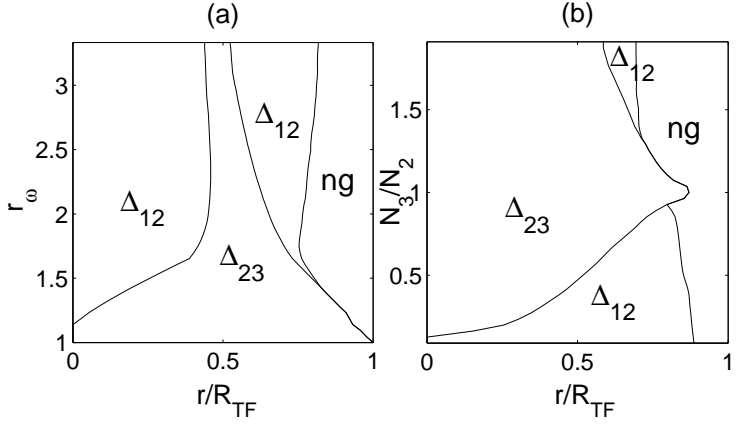

FIG. 2: (a) Zero temperature phase diagram as a function $r_{\omega}$ and $r / R_{T F}$ with particle numbers $N_{1,2,3}=5.5 \cdot 10^{4}$. (b) Zero temperature phase diagram as a function particle number ratio $N_{3} / N_{2}$ and $r / R_{T F}$, with $N_{1}=N_{2}=5.5 \cdot 10^{4}$ and $r_{\omega}=1$. In both Figures we used $k_{F} a_{12}=-1,\left(1+1 / r_{m}\right) k_{F} a_{23}=-1$, and $\left|a_{23}\right|>\left|a_{13}\right| . N$ indicates a normal gas or a vacuum at sufficiently large distances.

Barkhudarov 23] and we have generalized this calculation to deal with interactions between unequal mass fermions. We consider interacting atoms on the matched Fermi surfaces with energies equal to the chemical potentials since in the BCS regime physics close to the Fermi surface dominates. For the equal mass case the correction in the critical temperature is the usual $\approx 2.2$, but it increases as the second component becomes heavier. With $r_{m}=0.15$ the correction turns out to be $\approx 6.3$. Due to this mass dependence of the Gorkov correction, we expect that finite temperature mean-field calculations 
(a)

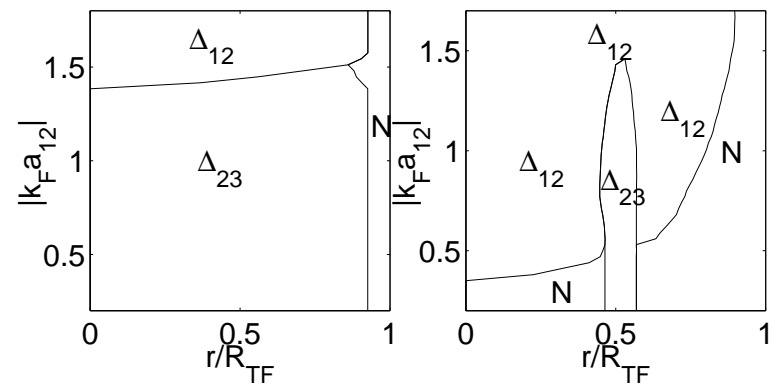

FIG. 3: Phase diagrams in the trap as functions $-k_{F} a_{12}$ and $r / R_{T F}$ for two different relative trap frequencies, in (a) $r_{\omega}=$ 1.0 and (b) $r_{\omega}=2.67$. We used parameters $N_{1,2,3}=5.5 \cdot 10^{4}$, $\left(1+1 / r_{m}\right) k_{F} a_{23}=1$, and $\left|a_{23}\right|>\left|a_{13}\right|$.

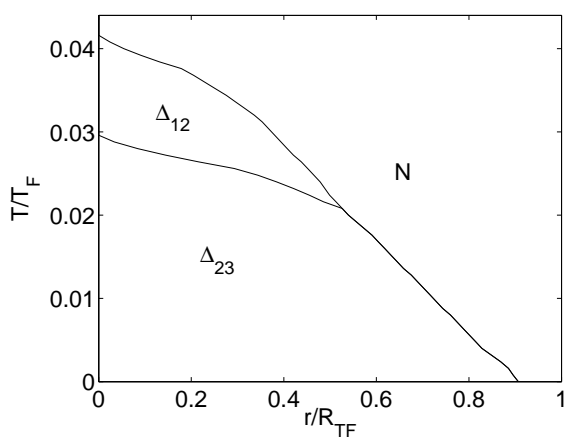

FIG. 4: Phase diagram as a function of the temperature $T$ and position. We used parameters $N_{1,2,3}=5.5 \cdot 10^{4}, r_{\omega}=$ $1, k_{F} a_{12}=-1,\left(1+1 / r_{m}\right) k_{F} a_{23}=-1$, and $\left|a_{23}\right|>\left|a_{13}\right|$. With our parameters the Fermi temperature $T_{F} \approx 600 \mathrm{nK}$ and $R_{T F} \approx 50 \mu \mathrm{m}$.

overestimate the critical temperature in the channel with unequal masses relative to the equal mass channel.

Conclusions.-We have solved the mean-field theory of a trapped three-component Fermi gas We found different superfluids coexisting in a same trapped system which can leave clear signals into the easily measurable density distributions.Such signatures of the spatial distribution of the different superfluids could be complemented by information about the different pairing gaps obtainable by rf-spectroscopy [5, 24]. With the our example parameters the coherence length in the center of the cloud turns out to be $0.11 \cdot R_{T F}$. While this is a fairly large fraction we nevertheless choose to use the local density approximation. This is unlikely to affect the general features of our results, which hinge on pairing with matched Fermi surfaces. By increasing the atom numbers the local density approximation becomes ever more accurate In this article we have ignored the surface energy contributions between different superfluids. However, by applying the results of De Silva and Mueller [25] we have estimated that in our case the surface contribution compared with the superfluid energy in the shell structures is at worst at the $2 \%$ level and usually much less than this. Therefore, they are unlikely to have a large effect on our phase diagrams. Furthermore, the surface energy compared with the superfluid bulk energy scales in such a way that it can always be made small by either increasing atom numbers or by reducing the trap frequency. It would be interesting to understand how co-existing superfluids of nontrivial spatial topology influence the collective and the rotational properties of these systems.

This work was supported by Academy of Finland (project numbers 207083, 106299, 205470) and conducted as part of a EURYI scheme award. See www.esf.org/euryi.

[1] C. Regal, M. Greiner, and D. Jin, Phys. Rev. Lett. 92, 040403 (2004).

[2] M. W. Zwierlein, C. A. Stan, C. H. Schunck, S. Raupach, A. Kerman, and W. Ketterle, Phys. Rev. Lett. 92, 120403 (2004).

[3] M. Bartenstein, A. Altmeyer, S. Riedl, S. Jochim, C. Chin, J. H. Denschlag, and R. Grimm, Phys. Rev. Lett. 92, 203201 (2004).

[4] J. Kinast, S. L. Hemmer, M. E. Gehm, A. Turlapov, and J. E. Thomas, Phys. Rev. Lett. 92, 150402 (2004).

[5] C. Chin, M. Bartenstein, A. Altmeyer, S. Riedl, S. Jochim, J. H. Denschlag, and R. Grimm, Science 305, 1128 (2004).

[6] M. W. Zwierlein, J. Abo-Shaeer, A. Schirotzek, C. Schunck, and W. Ketterle, Nature 435, 1047 (2005).

[7] R. Casalbuoni and G. Nardulli, Rev. Mod. Phys. 76, 263 (2004).

[8] M. W. Zwierlein, A. Schirotzek, C. H. Schunck, and W. Ketterle, Science 311, 492 (2006).

[9] G. B. Partridge, W. Li, R. I. Kamar, Y. Liao, and R. G. Hulet, Science 311, 506 (2006).

[10] M. W. Zwierlein, C. H. Schunck, A. Schirotzek, and W. Ketterle (2006), cond-mat/0605258.

[11] M. G. Alford and G. Cowan, J. Phys. G 32, 511 (2006).

[12] G. Roati, F. Riboli, G. Modugno, and M. Inguscio, Phys. Rev. Lett. 89, 150403 (2002).

[13] K. Günter, T. Stöferle, H. Moritz, M. Köhl, and T. Esslinger, Phys. Rev. Lett. 96, 180402 (2006).

[14] D. Petrov, C. Salomon, and G. Shlyapnikov, J. Phys. B: At. Mol. Opt. Phys 38, S645 (2005).

[15] M. Iskin and C. A. R. S. de Melo (2006), condmat/0604184.

[16] L. He, M. Jin, and P. Zhuang (2006), cond-mat/0604137.

[17] S.-T. Wu, C.-H. Pao, and S.-K. Yip (2006), condmat/0604572.

[18] C. Honerkamp and W. Hofstetter, Phys. Rev. Lett. 92, 170403 (2004).

[19] P. F. Bedaque and J. P. D'Incao (2006), condmat/0602525.

[20] T. Paananen, J.-P. Martikainen, and P. Törmä, Phys. Rev. A 73, 053606 (2006).

[21] L. M. Jensen, J. Kinnunen, and P. Törmä (2006), cond- 
mat/0604424.

[22] Y. Shin, M. W. Zwierlein, C. H. Schunck, A. Schirotzek, and W. Ketterle, Phys. Rev. Lett. 97, 030401 (2006).

[23] L. P. Gorkov and T. K. Melik-Barkhudarov, Zh. Eksp. Teor. Fiz 40, 1452 (1961).
[24] J. Kinnunen, M. Rodriguez, and P. Törmä, Science 305, 1131 (2004).

[25] T. N. D. Silva and E. J. Mueller, Phys. Rev. Lett. 97, 070402 (2006). 